\title{
Lost and found: applying network analysis to public health contact tracing for HIV
}

\author{
Dana K. Pasquale ${ }^{1,2^{*}} \mathbb{D}$, Irene A. Doherty ${ }^{3,4}$, Peter A. Leone ${ }^{5}$, Ann M. Dennis ${ }^{5}$, Erika Samoff ${ }^{6}$, Constance S. Jones ${ }^{6}$, \\ John Barnhart ${ }^{6}$ and William C. Miller ${ }^{7}$
}

*Correspondence:
dana.pasquale@duke.edu
1 Department of Sociology,
Duke University, 417 Chapel
Drive, 276 Soc/Psych
Building, Box 90088, Durham,
NC 27708-0088, USA
Full list of author information
is available at the end of the
article

*Correspondence: dana.pasquale@duke.edu 1 Department of Sociology, Drive, 276 Soc/Psych NC 27708-0088, USA article

\begin{abstract}
Infectious disease surveillance is often case-based, focused on people diagnosed and their contacts in a predefined time window, and treated as independent across infections. Network analysis of partners and contacts joining multiple investigations and infections can reveal social or temporal trends, providing opportunities for epidemic control within broader networks. We constructed a sociosexual network of all HIV and early syphilis cases and contacts investigated among residents of 11 contiguous counties in North Carolina over a two-year period (2012-2013). We anchored the analysis on new HIV diagnoses ("indexes"), but also included nodes and edges from syphilis investigations that were within the same network component as any new HIV index. After adding syphilis investigations and deduplicating people included in multiple investigations (entity resolution), the final network comprised 1470 people: 569 HIV indexes, 700 contacts to HIV indexes who were not also new cases themselves, and 201 people who were either indexes or contacts in eligible syphilis investigations. Among HIV indexes, nearly half ( $48 \% ; n=273$ ) had no located contacts during single-investigation contact tracing, though 25 (9\%) of these were identified by other network members and thus not isolated in the final multiple investigation network. Constructing a sociosexual network from cases and contacts across multiple investigations mitigated some effects of unobserved partnerships underlying the HIV epidemic and demonstrated the HIV and syphilis overlap in these networks.
\end{abstract}

Keywords: HIV-1, Sexual networks, Public health, Partner notification, Contact tracing, Syphilis, Epidemiology, North Carolina

\section{Introduction}

Several aspects of the network of relationships among which human immunodeficiency virus (HIV) diffuses can help guide intervention, including discerning the relationship ties (edges) along which the infection diffuses (Doherty et al. 2005), ascertaining mixing patterns and risk assortativity (Doherty et al. 2009, 2011; Schneider et al. 2013), and assessing the overlap of HIV and other sexually transmitted infections (STI) circulating within a socio-sexual network. HIV and syphilis, a bacterial infection, are both epidemics and in the southeastern United States (US).

Though HIV can be spread through behaviors such as sharing needles and syphilis can be transmitted during childbirth, in this region both share a population at risk and are author(s) and the source, provide a link to the Creative Commons licence, and indicate if changes were made. The images or other third party material in this article are included in the article's Creative Commons licence, unless indicated otherwise in a credit line to the material. If material is not included in the article's Creative Commons licence and your intended use is not permitted by statutory regulation or exceeds the permitted use, you will need to obtain permission directly from the copyright holder. To view a copy of this licence, visit http:// creativecommons.org/licenses/by/4.0/. 
primarily sexually transmitted. A single sexual act can transmit both infections. They are biologically synergistic, where being infected with one increases the possibility of acquiring the other infection (Augenbraun and McCormack 1994; Chesson et al. 1999; Buchacz et al. 2004; Karp et al. 2009; Mayer and Venkatesh 2011), and thus represent a complex public health problem (Fujimoto et al. 2018). Indeed, prior work has demonstrated that overlap of HIV and syphilis in sexual network components increases transmission risk (Fujimoto et al. 2018; Doherty et al. 2011; Cope et al. 2014).

HIV and syphilis are reportable diseases in all 50 states plus the District of Columbia (Institute of Medicine (US) Committee on the Ryan White CARE Act 2004), meaning that any healthcare provider or laboratory must report diagnoses to their governing public health body. Trained public health personnel meet with "index cases"-people who are newly diagnosed-to perform partner notification interviews and contact tracing for each new case of HIV and/or syphilis reported. During partner notification interviews, risk behaviors of the newly diagnosed index case are assessed and the case is asked to disclose sexual, needle-sharing, or social contacts who would benefit from sexually transmitted infection testing so that the public health personnel can trace the contacts. During contract tracing, these personnel locate these contacts, inform them of their potential risk, offer testing, and assist with treatment appointments if necessary. Though these person-based data lend themselves to network construction, infectious disease surveillance often focuses on individual cases and contacts, treating infected people and their traced contacts cross-sectionally and independently across infections. Network analysis of partners and contacts across investigations-both within and across multiple infections-can reveal social or temporal trends and provide opportunities for broader epidemic control (Liljeros et al. 2003), particularly among synergistic infections such as HIV and syphilis where decreasing the incidence of one infection may also decrease the incidence of the other infection.

In North Carolina (NC), as in other parts of the US South, young Black men disproportionately bear the burden of both HIV and syphilis (Sena et al. 2008; Centers for Disease Control and Prevention 2016, 2019). In North Carolina, Black men age 13 years and older make up less than one-quarter of the population (North Carolina HIV/STD/Hepatitis Surveillance Unit 2020b) yet accounted for approximately half (674/1383) (North Carolina HIV/STD/Hepatitis Surveillance Unit 2020a) of all new HIV diagnoses in 2019. As these young men are also less likely to have regular healthcare access (Krawczyk et al. 2006; Napravnik et al. 2006), public health contact tracing activities to locate, test, and treat them are critical. However, this mission is hampered by mistrust and social/structural issues which lead to incomplete contact ascertainment.

Contact tracing is name-based and conducted in NC by public health professionals called disease intervention specialists (DIS). DIS attempted to interview all persons newly diagnosed with HIV and/or early syphilis ("index cases") in the state. In these interviews, DIS elicit sexual partners, injecting drug use partners, and social contacts thought by the interviewee to possibly be at high risk of HIV exposure (Centers for Disease Control and Prevention 2008). The period of interest for which DIS elicit contacts during HIV investigations is the 12 months prior to diagnosis for established HIV cases; 6 months prior for persons thought to be recently infected based on acute viral illness or recent negative HIV test; and 2 months prior for persons diagnosed during acute HIV 
infection (AHI) per 4th generation antibody or RNA test results (Centers for Disease Control and Prevention 2014). The period of interest for syphilis is 3 months prior for primary syphilis, 6 months prior for secondary syphilis, and 12 months prior for early latent syphilis (Centers for Disease Control and Prevention 2014). For both infections, DIS also elicit and trace social contacts at their discretion since there appears to be an overlap between social networks and sexual partners (Brenner et al. 2011), particularly among Black men who have sex with men (MSM) (Schneider et al. 2013; Tieu et al. 2015). Upon elicitation, DIS determine whether there is enough information on each contact to begin the process of attempting to trace them ("investigation"); contacts without enough locating information are deemed "marginal" partners and not traced, though marginal partners can revert to investigated partners if more locating information is uncovered. Contacts with sufficient locating information are "initiated" for tracing and testing. DIS then investigate the initiated contacts, which includes a records search in the database and/or an attempt to locate the contact for testing and interview. All cooperation with contact tracing is voluntary.

We sought to augment information gleaned from individual HIV investigations in central NC by combining multiple HIV investigations and adding information from early syphilis investigations into a single network. We then described where and how consideration of the broader network added context through understanding component composition that would not be known by treating each case independently. Finally, we assessed the overlap between HIV and syphilis circulating among network components constructed from disclosed contacts.

\section{Methods}

\section{Study population, setting, and data}

NC is divided into 10 regions for HIV and sexually transmitted disease (STD) control activities. Region 6 in north central NC (Fig. 1) comprises seven metropolitan and four non-metropolitan counties based on 2013 US Department of Agriculture rural-urban continuum codes (USDA Economic Research Service 2013), with a total population in 2013 of 1.9 million persons including 8,700 persons living with HIV (North Carolina HIV/STD/Hepatitis Surveillance Unit 2015b). The rate of new HIV diagnoses in Region 6 was 16.3 per 100,000 population in 2013, corresponding to 315 new diagnoses (North Carolina HIV/STD/Hepatitis Surveillance Unit 2015b). Black men who have sex with men (MSM) were disproportionately affected, accounting for nearly half of all HIV diagnoses among men (North Carolina HIV/STD/Hepatitis Surveillance Unit 2015a).

We abstracted infectious disease surveillance and linked index-and-contact public health contact tracing data elicited during partner notification for HIV and syphilis diagnoses made among residents aged $\geq 14$ years of the 11 contiguous counties in Region 6 over a two-year period (2012-2013). We matched the named cases and contacts across all contact tracing investigations conducted in these counties during the two-year period. Some contacts were diagnosed as part of contact tracing activities (i.e., as contacts of newly diagnosed cases) and were then also considered to be an index if they were diagnosed during 2012-2013 while residing in one of the 11 counties under study. As entering these sociosexual networks with high HIV prevalence is associated with future HIV acquisition among Black MSM (Hurt et al. 2012), we also checked for 


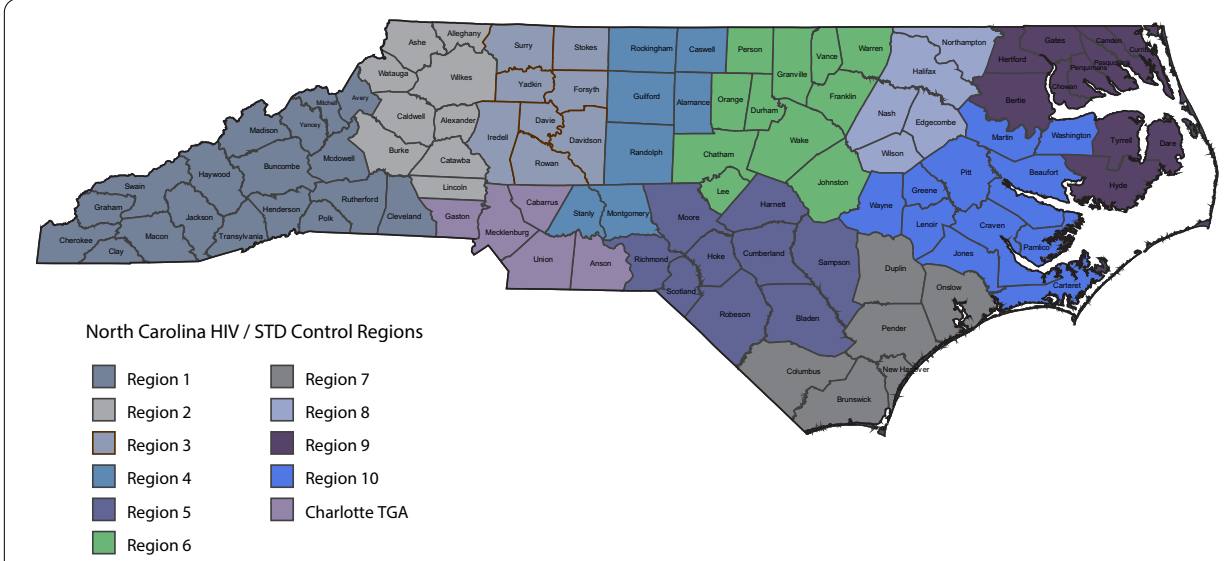

Fig. 1 North Carolina Prevention and Care Services Regions. Legend: Map showing the North Carolina Regions for Prevention and Care Services. We abstracted HIV and syphilis cases diagnosed during 2012-2013 from among residents of the 11 contiguous counties of Region 6 (green counties)

new HIV diagnoses made through 2018 among network members who were not known to be HIV-positive during the 2012-2013 study period.

\section{Sociosexual network construction}

Contact tracing and partner notification data collected by DIS during HIV and syphilis investigations were used to create an undirected sociosexual network of disclosed ties using the igraph (Csárdi and Nepusz 2006) package in R (R Core Team. R 2015). Nodal attributes collected via self-report included demographic characteristics (ethnicity/ race, five-year age category, gender/sexual orientation, county of residence), risk behaviors (drug use, condom use, sexual relationships with people known to be HIV-positive, partnership dynamics), and HIV and STD testing and infection history. Edge attributes included the type of relationship (sexual, social, needle-sharing) and the infection being investigated.

We created the edgelist from relationships elicited across multiple HIV and syphilis case investigations. All network members were de-identified after edgelist construction to preserve confidentiality. All contacts disclosed by newly diagnosed HIV or syphilis cases in this geographic area were used to construct the edgelist and are represented in the network, even if the relationships were not concurrent. We have several reasons for constructing the network in this way: young men who enter these components frequently remain linked into them and go on to acquire HIV at higher rates (Hurt et al. 2012); both HIV and syphilis circulating among the same group carries a potential risk for both infections even if partnerships are not concurrent; and HIV is a lifelong infection. While transmission can be prevented through condom use and antiretroviral therapy to reduce viral load and thus infectiousness, nearly 1 in 3 HIV indexes in this set $(n=180)$ failed to achieve viral suppression within three years of diagnosis, with Black men less likely than men of other races, and another $11 \%(n=65)$ had inconsistent viral suppression during the three-year post-diagnosis period. Thus, contacts which occurred after the HIV index's diagnosis still represent a pathway to consider for infection 
diffusion given that at least one of the nodes would be HIV-positive and some of the components also had circulating syphilis.

We compiled the network from the edgelist. Our primary focus was HIV index case investigations; partnerships elicited during syphilis investigations in Region 6 during 2012-2013 were only included to better understand network connectivity among persons at risk of HIV. As such, syphilis network components that did not include at least one HIV case were excluded from the analysis network since they would not affect network metrics calculated with respect to HIV indexes. The University of North Carolina Biomedical Institutional Review Board approved the study.

\section{Descriptive network analyses}

Improvements in network ascertainment and changes in connectivity were measured at each step of DIS partner services activities where a case might have no traced partners yet was identified by another network member. We compared network structures between indexes who disclosed contacts who were initiated for partner services to indexes who did not have any outgoing contacts initiated but who were identified by another network member and so were not isolated in the final observed network. We assessed how network construction can provide additional context to HIV investigations as measured by a decrease in the number of HIV indexes with no known contacts and reveal the overlap.

The network immediately surrounding each HIV index was treated as a local ego network and network characteristics and structures ( $k$-cores, triangles) were calculated on the basis of index network position. We measured component sizes and distribution, component composition by sociodemographic characteristics and infection type, and bridging of HIV investigation edges by syphilis investigation edges determined from separate contact tracing efforts.

\section{Results}

\section{Study population}

During the two-year study period (2012-2013), 569 new HIV index diagnoses were reported among residents aged $\geq 14$ years across the eleven Region 6 counties under study; these HIV indexes formed the core of the sociosexual network analyzed. Most were male (79\%) and non-Hispanic Black (66\%). Median age at diagnosis was 33 years (IQR: 24-45) (Table 1). Laboratory results indicated that 32 (6\%) were acutely or recently infected with HIV and 144 (25\%) had already progressed to acquired immunodeficiency syndrome (AIDS) by the time of diagnosis.

\section{Contact tracing}

DIS interviewed nearly all indexes (97\%), although $26 \%$ indexes $(n=146)$ declined to discuss or provide names of contacts. The $74 \%$ who disclosed contacts $(n=423)$ reported a total of 1,850 sexual partners (median $=2$ (IQR: $1-4$ ), range $0-60), 130$ social contacts (range 0-19), and 5 needle-sharing partners (range $0-3$ ) in the 2, 6, or 12 months prior to diagnosis (depending on infection stage). Of the 1,850 sexual partners reported, 521 (28\%) did not have enough locating information to initiate partner notification. 


\section{Relationships with investigated contacts}

DIS ultimately succeeded in documenting 845 relationships for notification and testing: 749 sexual partnerships, 92 social contacts, and 4 needle-sharing partnerships, representing $40 \%, 71 \%$, and $80 \%$ of total partnerships/contacts reported, respectively. These relationships composed the sociosexual network edges. Indexes with at least one investigated contact had a median of 2 first-degree network contacts (IQR:1-3, range: 1-25). Most sexual partnerships were among people of the same race (78\%), included at least one person of Black race (77\%), and were between two men (72\%). In half (51\%) of sexual partnerships, partners were within five years of age of each other. Among the 749 sexual and 4 needle-sharing partners, 42\% (319/753) were also HIV-infected including some who diagnosed as indexes themselves during the study period 2012-2013 (diagnosis year range 1995-2017, 46\% diagnosed $\geq 6$ months prior to index), 30\% of contacts tested HIV-negative during the investigation, and $27 \%$ had unknown serostatus.

\section{Sociosexual network population}

All indexes and any contacts investigated were included in the network. After de-duplicating people who appeared in multiple investigations (entity resolution: 106/845 contacts were indexes themselves and the other 39 were contacts of $>1$ index), 700 unique first-degree HIV contacts were added to the network in addition to the 569 indexes. After de-duplication, the syphilis investigations added 201 more people who were in the same component as at least one HIV index. The final total network size was 1470 persons (Fig. 2a) and included 569 newly-diagnosed HIV indexes plus 901 network members who were observed to be first-degree contacts of an HIV index $(n=700)$ or indirectly linked to an HIV index in the same sociosexual network component $(n=201)$, while not being HIV index cases themselves. Of these 901, 283 (31\%) network members were HIV-positive (median diagnosis year 2009 (IQR: 2006-2012) excluding 80 with unknown diagnosis years), 272 (30\%) were HIV negative based upon a test during the investigation period, and serostatus was unknown for the remaining 346 (38\%) due to inability to locate the contact upon investigation or the contact refusing counseling and/ or current testing upon location.

\section{Sociosexual network construction and composition}

Despite interviewing $97 \%(\mathrm{n}=551)$ of HIV indexes, nearly half $(48 \%, 273 / 569)$ had no located contacts during their individual contact tracing investigation. After adding syphilis investigation information and creating a network, 25 of 273 (9\%) HIV indexes without any traced contacts were identified by other network members and thus were not isolated in the final observed network ( $\mathrm{n}=248$ remained isolated with no located contacts).

Nearly one-quarter (128/569) of indexes did not disclose partners, though $10(8 \%)$ of these 128 were then identified as a partner by another investigated HIV or syphilis case, so losses at that step in the DIS interview process contributed 118 rather than 128 of the 248 isolates (Fig. 3). In other words, 10 indexes who refused to disclose contacts had at least one network link and were not isolated because they were identified by another network member. The next largest loss was 56/569 (10\%) indexes 
Table 1 Index HIV cases aged 14 years and older diagnosed 2012-2013 in NC HIV/STD Control Region 6 and their disclosed and traced first-degree contacts in the sociosexual network ( $N=1269)$

\begin{tabular}{|c|c|c|c|c|}
\hline & \multicolumn{2}{|c|}{ Index $(n=569)$} & \multicolumn{2}{|c|}{ Contact $(n=700)$} \\
\hline & $n$ & (\%) & $\mathrm{n}$ & (\%) \\
\hline \multicolumn{5}{|l|}{ Gender } \\
\hline Male & 451 & (79) & 581 & (83) \\
\hline Female & 114 & $(20)$ & 98 & $(14)$ \\
\hline Transgender (M to F) & 4 & $(0.7)$ & 0 & - \\
\hline Not indicated & 0 & - & 21 & (3) \\
\hline \multicolumn{5}{|l|}{ Race/Ethnicity } \\
\hline Non-Hispanic White & 114 & $(20)$ & 164 & (23) \\
\hline Non-Hispanic Black & 378 & (66) & 459 & $(66)$ \\
\hline Hispanic, any single race & 58 & (10) & 33 & (5) \\
\hline Other or mixed race & 19 & (3) & 31 & (4) \\
\hline Not indicated & 0 & - & 13 & $(2)$ \\
\hline \multicolumn{5}{|l|}{ Region of birth } \\
\hline USA-50 states & 530 & (93) & 344 & $(49)$ \\
\hline $\begin{array}{l}\text { Latin / South America, Caribbean (incl. US } \\
\text { Territories) }\end{array}$ & 24 & (4) & 6 & $(0.9)$ \\
\hline Europe, Asia, Oceania & 3 & $(0.5)$ & 2 & $(0.3)$ \\
\hline Africa & 12 & $(2)$ & 0 & - \\
\hline Not indicated & 0 & - & 348 & $(50)$ \\
\hline \multicolumn{5}{|l|}{ Marital status } \\
\hline Currently married & 39 & (7) & 44 & (6) \\
\hline Divorced / separated / widowed & 20 & (4) & 9 & (1) \\
\hline Never married & 413 & (73) & 413 & (59) \\
\hline Not indicated & 97 & (17) & 234 & (33) \\
\hline \multicolumn{5}{|l|}{ County of residence } \\
\hline Urban & 437 & (77) & -- & \\
\hline Suburban & 27 & (5) & -- & \\
\hline Rural & 105 & (18) & -- & \\
\hline \multicolumn{5}{|l|}{ Age at index case's HIV diagnosis (years) ${ }^{\mathrm{a}}$} \\
\hline$\leq 19$ & 29 & (5) & 46 & (7) \\
\hline $20-29$ & 214 & (38) & 316 & (45) \\
\hline $30-39$ & 101 & (18) & 169 & $(24)$ \\
\hline $40-49$ & 136 & (24) & 88 & (13) \\
\hline$\geq 50$ & 89 & $(16)$ & 52 & (7) \\
\hline Not indicated & 0 & -- & 29 & (4) \\
\hline Median (IQR) & 33 & $(24-45)$ & 28 & $(23-37)$ \\
\hline \multicolumn{5}{|l|}{ HIV status } \\
\hline Positive & 569 & $(100)$ & 221 & $(35)$ \\
\hline Negative & -- & & 243 & $(32)$ \\
\hline Unknown & - & & 236 & (34) \\
\hline Year of HIV diagnosis & & & $n=221$ & \\
\hline$<2006$ & - & & 50 & (23) \\
\hline 2006-2010 & - & & 71 & $(32)$ \\
\hline 2011 & - & & 21 & $(10)$ \\
\hline 2012 & 271 & (48) & 13 & (6) \\
\hline 2013 & 298 & (52) & 7 & (3) \\
\hline$\geq 2014$ & - & & 35 & (16) \\
\hline Not indicated & - & & 24 & $(11)$ \\
\hline
\end{tabular}


Table 1 (continued)

\begin{tabular}{|c|c|c|c|c|}
\hline & \multicolumn{2}{|c|}{ Index $(n=569)$} & \multicolumn{2}{|c|}{ Contact $(n=700)$} \\
\hline & $\mathrm{n}$ & (\%) & $n$ & (\%) \\
\hline \multicolumn{5}{|l|}{ HIV stage at diagnosis ${ }^{\mathrm{b}}$} \\
\hline Acute/recent & 32 & (6) & - & \\
\hline Chronic, non-AIDS & 393 & (69) & - & \\
\hline Chronic, AIDS & 144 & (25) & - & \\
\hline
\end{tabular}

${ }^{a}$ Among partners, for earliest record associated with any index case

${ }^{\mathrm{b}}$ Based upon laboratory results

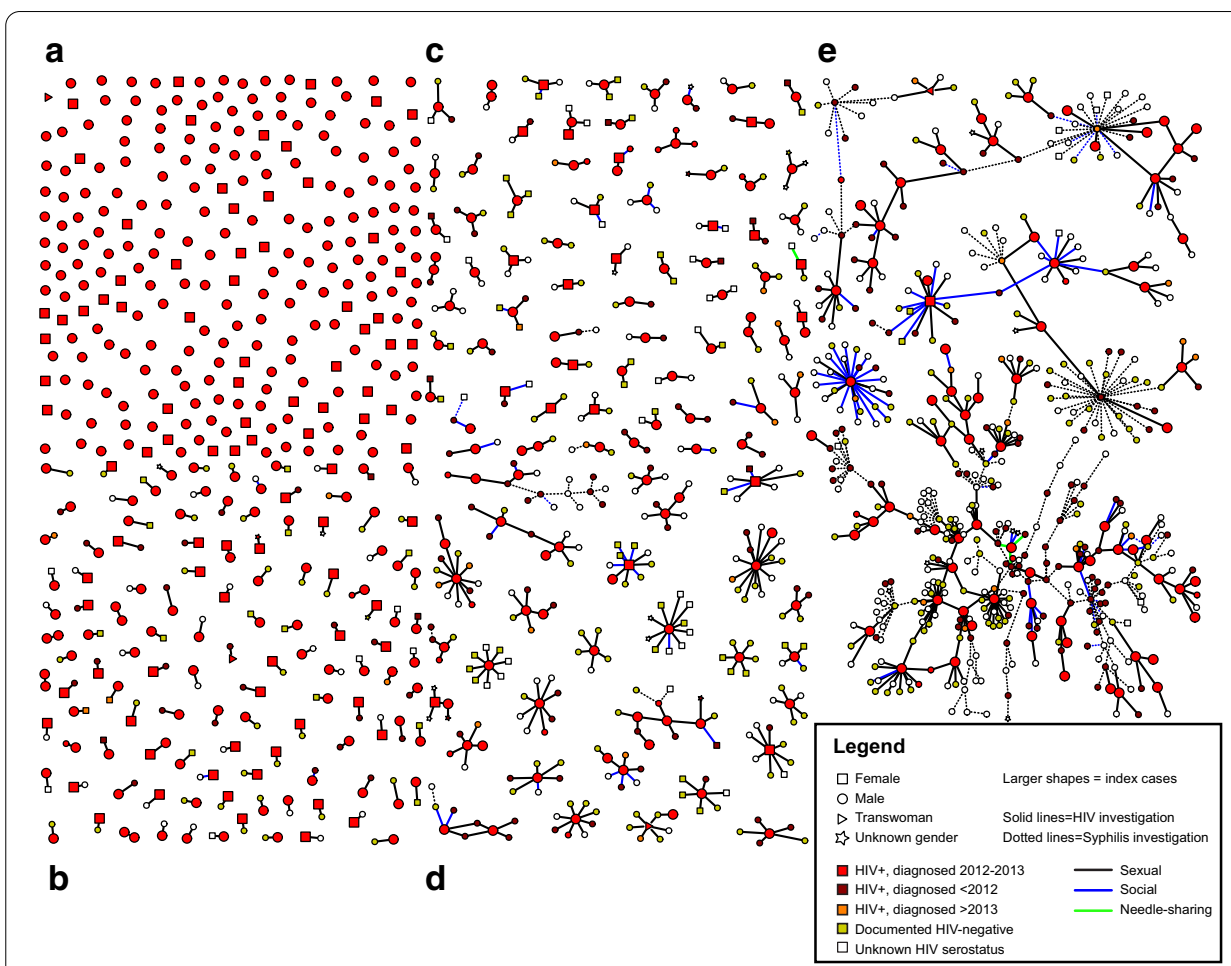

Fig. 2 a. Sociosexual network $(N=1470)$. Sociosexual network showing 569 index cases newly diagnosed with HIV in the area around Raleigh, North Carolina, during 2012-2013. Total graph includes 1470 persons distributed in 468 network components. Graph shows gender (node shape), HIV status (node color), HIV index case status (node size), type of contact (edge color), and whether the contact was part of an HIV or syphilis investigation (edge thickness). Graph is loosely grouped by size of sociosexual network component: a isolates $(n=248$ people), $\mathbf{b}$ dyads ( $n=238$ people distributed across 119 components), $\mathbf{c}$ components size 3-4 ( $n=224$ people distributed across 68 components), $\mathbf{d}$ components size $5-16(n=241$ people distributed across 29 components), and e) components size $26,81,92$, and 320 ( $n=519$ people distributed across 4 components). b. Three largest components of the sociosexual network $(n=493)$. Legend: Sociosexual network showing the three largest components representing 493/1,470 (34\%) network members (from left to right, 320, 92, and 81 people, respectively). Graph shows gender (node shape), HIV status (node color), index case status (node size), type of contact (edge color), and whether the contact was part of an HIV or syphilis investigation (edge thickness). The middle component ( $n=92$ nodes) would have been observed as six smaller HIV investigation components (indicated by gray background) without inclusion of the syphilis investigation partnerships bridging the relationships elicited during the HIV investigations 


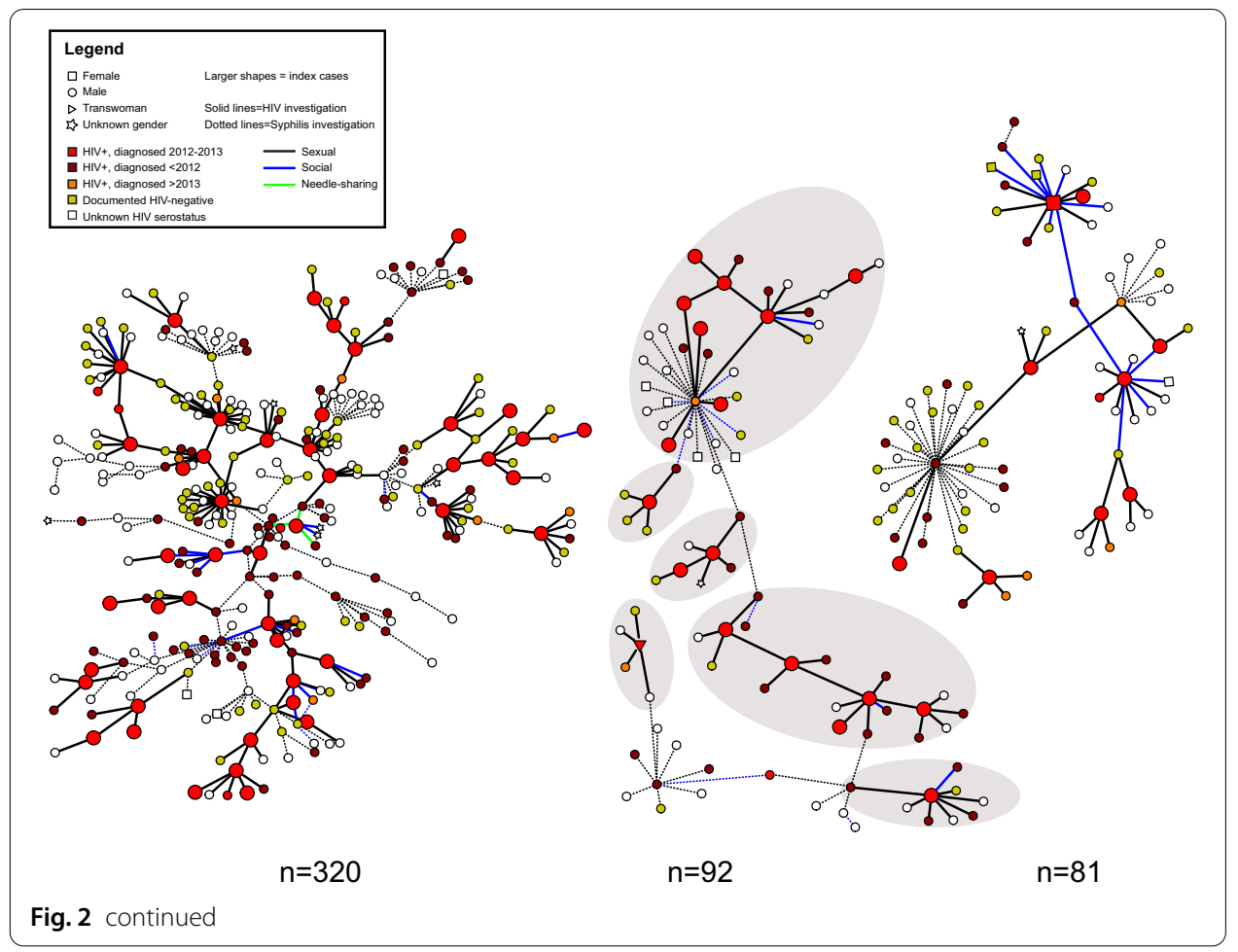

whose partners were investigated, though none could be located; however, 7 of 56 (13\%) were identified by another network member. Along all of the partner services steps, linkages from other cases in the network "found" the highest proportion among indexes who only had partners without sufficient information to begin tracing (18\%, $5 / 28)$.

Among all 248 indexes who remained isolated after allowing for linkages from other cases and contacts in the network, $54 \%(\mathrm{n}=134)$ did not disclose partners because they were not located or refused, $17 \%(\mathrm{n}=42)$ reported zero sexual partnerships, and $29 \%$ $(\mathrm{n}=72)$ reported $1-50$ partners (median $=2$, IQR: $1-4)$ though none could be located (Fig. 3). However, 20/42 (48\%) indexes who reported zero partners and were not identified by another network member were concurrently diagnosed with HIV and AIDS (late stage disease), and may not have had any partners to elicit in the 12 months prior to diagnosis (the interview window) (Hollingsworth et al. 2008).

Besides the 248 isolates, the remaining 321 indexes formed 220 discrete components of $\geq 2$ people, most of which contained two people ( $\mathrm{n}=238$ people across 119 dyads) or three people ( $\mathrm{n}=144$ people across 48 components) when using both the HIV and syphilis investigations (Fig. 2a). The largest component included 320 persons (22\% total network, $8 \%$ (46/569) indexes). Component size, dominant demographic characteristics, and member HIV status are shown in Tables 2 and 3. Among non-isolated indexes, 7 (1\%) were in at least one triangle and 22 were in a $k$-core, comprising $21(4 \%)$ in a 2 -core and $1(0.2 \%)$ in a 3 -core.

When restricting to the 321 non-isolated indexes, those who disclosed contacts which were initiated $(n=296)$ did not differ in terms of age, ethnicity/race, gender, or sexual preference from indexes who did not have any initiated contacts of their own but who 

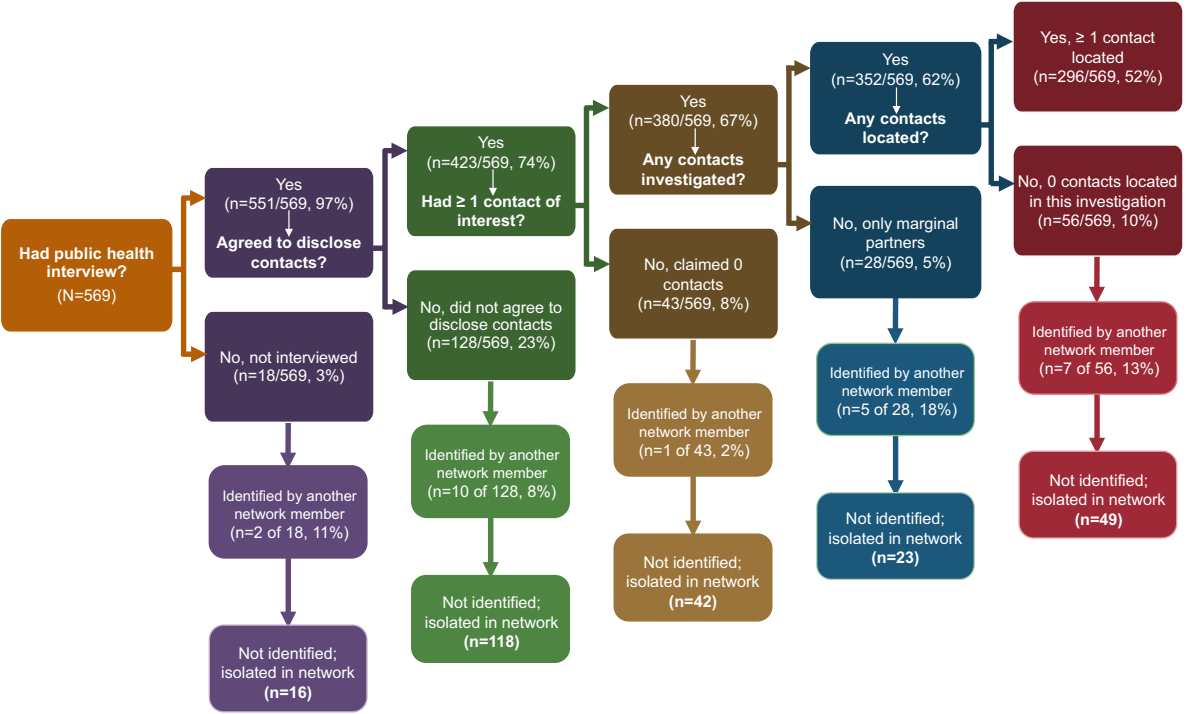

Fig. 3 Public health interview continuum and network isolation. This figure shows the progress along the disease intervention specialist (DIS) interview continuum leading to isolation in the network. Upon receiving a positive test, the index case is contacted for public health interview. The interview has two parts, an assessment of risk and partner elicitation for contact tracing by a DIS; the index can refuse to disclose contacts. If the index agrees to disclose contacts then the DIS conducting the interview elicits all contacts who may be the source of infection or who may have acquired the infection from the index ("source" and "spread"). A DIS then determines whether there is enough identifying information on contacts to initiate the tracing process and attempts to locate contacts who have sufficient information so that they may be offered testing. Contact tracing for an index case can end at any point in this process, resulting in an index case having zero traced contacts. This chart depicts the contact tracing steps, the end result of contact tracing for each HIV index case ( $N=569)$, and where constructing the network added context to HIV index cases with zero of their own traced partners by showing where the HIV index cases with zero of their own traced contacts were identified as persons of interest in HIV or syphilis contact tracing investigations conducted during the same time period in the same geographic area. Nearly half (273/569, 48\%) of HIV indexes had no located contacts during contact tracing. However, by linking HIV and syphilis investigations from the same time period and area, 25 of these 273 (9\%) indexes were identified by another network member which adds context to the local HIV epidemic and permits public health personnel to better understand transmission patterns

were identified by another network member $(n=25)$. However, indexes who did not have any initiated contacts of their own were more likely to be in a large component. Though a similar proportion were in a component size $2(10 / 25,40 \%$, vs. $115 / 296,39 \%)$, indexes who did not have any initiated contacts were significantly more likely to be in one of the 3 largest components $\left(44 \%\right.$ vs. $\left.21 \%, \chi^{2}(1, N=321)=7.0, p<0.01\right)$.

\section{Bridging of HIV by syphilis investigations}

Ten (4\%) of 220 components contained a mix of partnerships collected during syphilis investigations which encompassed overlapping people, with between 1 and 137 syphilis partnerships / edges (median $=1.5$, IQR:1-36) included in the component (Tables 2 and 3). Median size of the 10 components containing HIV and syphilis investigation partnerships was 11 persons (IQR: 4-81, range: 3-320), including 2 HIV indexes (median, IQR:1-9, maximum $=46$ ) and 0-22 syphilis indexes (median =0, IQR: 0-2). Persons involved in syphilis investigations accounted for $11-63 \%$ of these components $($ mean $=32 \%, S D=15 \%)$. The 3 largest of the 10 components $(n=81,92$, and 320 


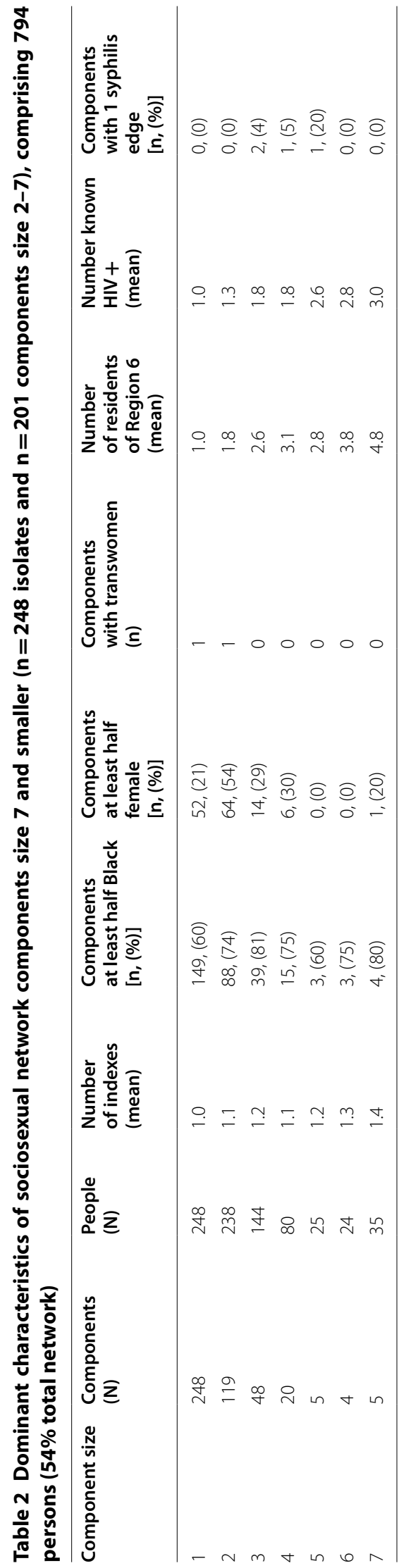




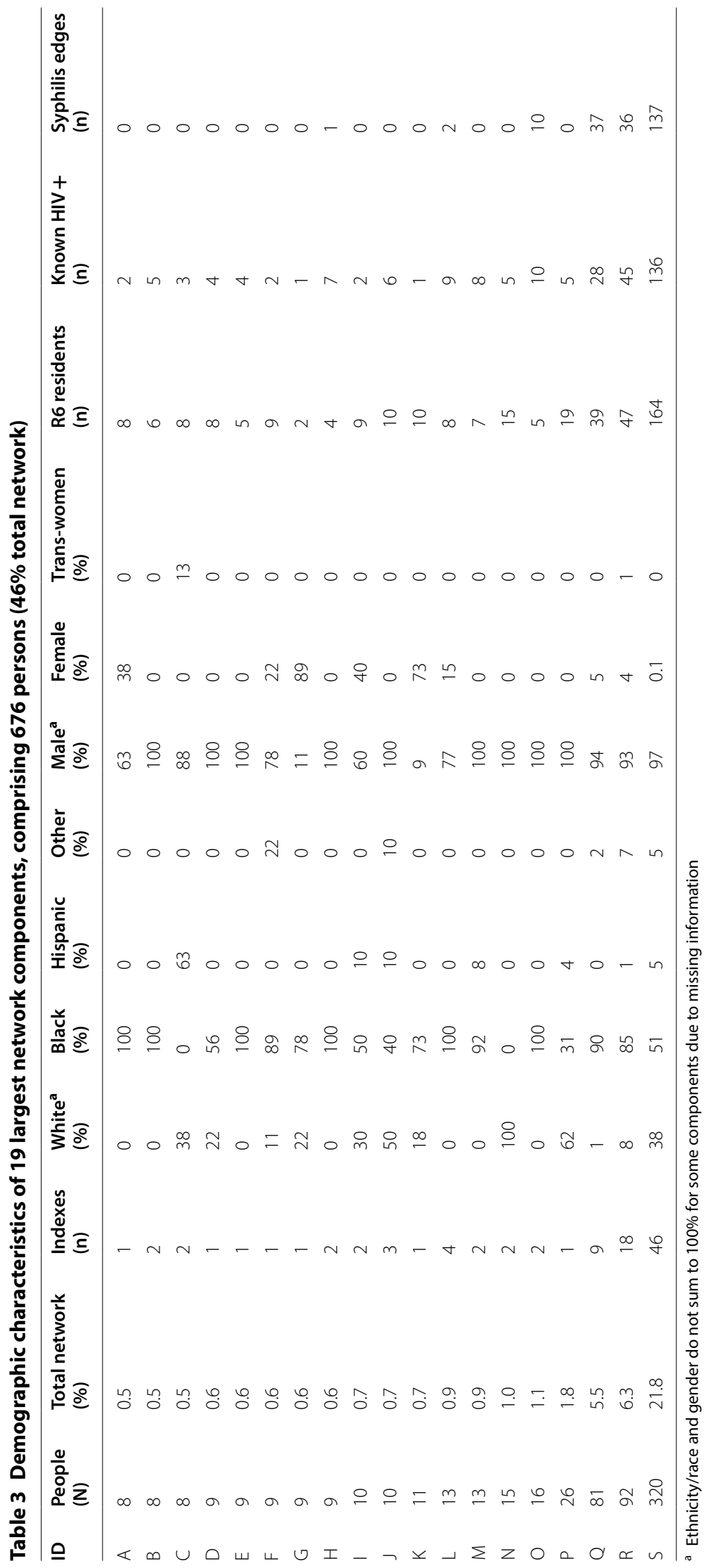


people) (Fig. 2b) included two or more HIV indexes bridged by partnerships elicited during syphilis investigations and would have appeared to be several smaller, separate components without the syphilis information (see component sized 92 in Fig. 2b for an example). In the component $n=320$, several closed loops included a mix of HIV and syphilis investigation partnerships. Among 2012-2013 investigations contributing to these 10 components, 74 were indexes in HIV investigations only and 63 were indexes in a syphilis investigation (may or may not have been an HIV index as well). Cases who were only HIV indexes were more likely to not participate in contact disclosure (14\%) compared to cases who were involved in syphilis only or syphilis and HIV investigations $(3 \%, p=0.04)$. Among indexes who did disclose contacts, there was no significant difference by infection in the average number of contacts per month across the period of interest.

\section{Post-study period HIV diagnoses}

Of 221 first-degree partners of HIV index cases who were documented to be HIV-positive, 35 (16\%) were diagnosed with HIV following the 2012-2013 investigation period during 2014-2018 (32 during 2014-2016 and 3 during 2017-2018) (Table 1). Half (17/35) had evidence of a negative HIV test result which was collected 2012-2013 and reported to the surveillance database, typically collected $2+$ years prior to HIV diagnosis date (median 2.2 years, IQR:1.7-3.5, range:1.3-3.9 years). The other 18 did not have a record of a 2012-2013 HIV test result: 3 refused, 3 couldn't be located at that time, 1 noted a current negative result though the laboratory result was not present in the database, 2 noted negative test results from prior to the study period which were not repeated, 5 no indication, and 4 had only the future positive test recorded. One in the last category was a sexual partner without current or recent negative HIV test results who was diagnosed in early 2014 as a result of partner tracing of an index case in this analysis.

Of the 201 network members who were part of a syphilis investigation and not firstdegree partners to an HIV index case, 139 were initiated for partner services and not noted to be HIV-positive during the 2012-2013 study period (29 documented HIV negative and 110 unknown HIV status). Of these 139, 9 (6\%) had evidence of 2014-2016 HIV diagnoses in the surveillance database and at least 4 (3\%) were diagnosed in 2017 and 2018. All 13 were in the 3 largest components constructed from the 2012-2013 investigations, which were predominantly MSM and included both HIV and syphilis investigations.

\section{Discussion}

Contact tracing is a key component of public health for infectious disease control of HIV and syphilis. Many challenges affect data quality and completeness during contact tracing and partner notification services, which can dampen service effectiveness. By incorporating information from multiple DIS investigations and examination of cases and their contacts in a more holistic way, sociosexual network analysis can mitigate some of the effects of unobserved partnerships underlying the HIV epidemic. Upon constructing the network, we were able to glean information about cases whose partners were not disclosed or located, which places those cases in a risk context related to the structure and composition of the components into which they were linked. That indexes who did not disclose partners but were identified by another network member were more likely 
to appear in larger components than people who did disclose located partners merits further investigation.

A median time of two years between negative and positive HIV tests among the HIV contacts who were later diagnosed is an indicator that behaviors sufficient for HIV acquisition continued even after there was an opportunity for public health intervention. Identifying the correlates of risk in a broader context can distinguish where preventive efforts should be directed. The overlap between HIV and syphilis in the larger components is consistent with prior research (Juher et al. 2017) and with clinical guidelines directing preventive HIV treatment (pre-exposure prophylaxis, or PrEP) evaluations toward people diagnosed with syphilis, further supported by the apparent subsequent acquisition of HIV in some of the syphilis network members. HIV and syphilis share at-risk populations and a primary mode of transmission. One limitation of the reportable disease mandate is that lab tests for HIV and syphilis with negative results are not reportable. Thus, we were not able to document negative HIV status for the majority of the second-degree syphilis investigation network members and some may have been HIV-positive during the study period but were not tested.

All 13 of the syphilis investigation members who tested positive for HIV after the study period were in large components with both HIV and syphilis circulating widely. This suggests that inclusion in one of these large components is an excellent indicator that preexposure prophylaxis (PrEP) to protect against HIV is warranted, particularly if the person has a recent sexually transmitted bacterial infection. Beyond an evaluation for PrEP, directing additional resources toward supporting an ongoing patient-provider relationship or subsidizing a prescription for PrEP for men who are part of these large components with circulating HIV and syphilis might have the benefit of averting cases, which can have a network-wide effect (Jenness et al. 2016).

As the largest components would have appeared to be smaller components without including information from the syphilis investigations, the inclusion of these edges revealed a more accurate picture of the underlying sociosexual network than would have been observed from the HIV investigations alone. Sociosexual networks provide an opportunity for intervention to reduce HIV transmission by adding context to public health contact tracing investigations so that limited resources can be directed toward areas of uncontrolled HIV or risk behaviors which have the potential for transmission.

\section{Abbreviations}

AHI: Acute HIV infection; AIDS: Acquired immunodeficiency syndrome; DIS: Disease investigation specialist; HIV: Human immunodeficiency virus; IQR: Interquartile range; MSM: Men who have sex with men; NC: North Carolina; PrEP: Pre-exposure prophylaxis; RNA: Ribonucleic acid; SD: Standard deviation; STD: Sexually transmitted disease; USA: United States.

\section{Acknowledgements}

We would like to thank the NC Department of Health and Human Services and the disease intervention specialists serving NC Prevention and Care Services Region 6.

\section{Authors' contributions}

DKP, PAL, and WCM conceived of the presented idea. DKP abstracted the data, created the sociosexual network, conducted the analyses, and was primary author of the manuscript. IAD, PAL, AMD, and WCM supervised the findings of this work. IAD, ES, CSJ, and JB are subject matter experts who guided the data abstraction and interpretation. IAD and AMD contributed to the final version of the manuscript. All authors discussed the results; provided critical feedback; and helped shape the research, analysis, and manuscript. All authors read and approved the final manuscript.

\section{Funding}

The project described was supported by the NIH T32 Grant Award 5T32Al070114-10 to UNC-Chapel Hill. DKP was also supported by the Eunice Kennedy Shriver National Institute of Child Health and Human Development (NICHD), NIH, 
Grant Award 1R25HD079352-01A1. AMD was supported by the National Institute of Allergy and Infectious Diseases (NIAID), NIH, through Grant Award Number K08Al112432. IAD was supported with supplemental funds from the National Center for Advancing Translational Sciences (NCATS), NIH, and RTI through Grant Award Number UL1TR001111.

\section{Availability of data and materials}

The datasets generated and/or analyzed are not publicly available. This analysis uses public health contact tracing and surveillance data, and is thus considered protected health information.

\section{Competing interests}

No competing interests or conflicts of interest are declared. The content is solely the responsibility of the authors and does not necessarily represent the official views of the $\mathrm{NIH}$ or the North Carolina Department of Health and Human Services.

\section{Author details}

'Department of Sociology, Duke University, 417 Chapel Drive, 276 Soc/Psych Building, Box 90088, Durham, NC 27708-0088, USA. ${ }^{2}$ Department of Epidemiology, Gillings School of Global Public Health, University of North Carolina At Chapel Hill, Chapel Hill, NC, USA. ${ }^{3}$ RTI International, Research Triangle Park, Durham, NC, USA. ${ }^{4}$ The Julius L. Chambers Biomedical Biotechnology Research Institute, North Carolina Central University, Durham, NC, USA. ${ }^{5}$ Division of Infectious Diseases, School of Medicine, University of North Carolina at Chapel Hill, Chapel Hill, NC, USA. ${ }^{6}$ NC Department of Health and Human Services Communicable Disease Branch, Raleigh, NC, USA. ${ }^{7}$ Division of Epidemiology, College of Public Health, The Ohio State University, Columbus, OH, USA.

Received: 27 October 2020 Accepted: 25 January 2021

Published online: 17 February 2021

\section{References}

Augenbraun MH, McCormack WM (1994) Sexually transmitted diseases in HIV-infected persons. Infect Dis Clin North Am 8(2):439-448

Brenner BG, Roger M, Stephens D, Moisi D, Hardy I, Weinberg J et al (2011) Transmission clustering drives the onward spread of the HIV epidemic among men who have sex with men in quebec. J Infect Dis 204(7):1115-1119

Buchacz K, Patel P, Taylor M, Kerndt PR, Byers RH, Holmberg SD et al (2004) Syphilis increases HIV viral load and decreases CD4 cell counts in HIV-infected patients with new syphilis infections. AIDS 18(15):2075-2079

Centers for Disease Control and Prevention (2008) Recommendations for partner services programs for HIV infection, syphilis, gonorrhea, and chlamydial infection. MMWR 57(RR-9)

Centers for Disease Control and Prevention (2014) Partner services providers quick guide. National Center for HIV/AIDS, Viral Hepatitis, STD, and TB Prevention, U.S. Department of Health and Human Services, Atlanta, GA

Centers for Disease Control and Prevention (2016) HIV in the southern United States, in CDC Issue Brief. National Center for HIV/AIDS Viral Hepatitis, STD, and TB Prevention, U.S. Department of Health and Human Services, Atlanta, GA

Centers for Disease Control and Prevention (2019) Sexually transmitted disease surveillance 2018, U.S. Department of Health and Human Services: Atlanta, GA. https://doi.org/10.15620/cdc.79370

Chesson HW, Pinkerton SD, Irwin KL, Rein D, Kassler WJ (1999) New HIV cases attributable to syphilis in the USA: Estimates from a simplified transmission model. AIDS 13(11):1387-1396

Cope AB, Crooks AM, Chin T, Kuruc JD, McGee KS, Eron JJ et al (2014) Incident sexually transmitted infection as a biomarker for high-risk sexual behavior after diagnosis of acute HIV. Sex Transm Dis 41(7):447-452

Csárdi G, Nepusz T (2006) The igraph software package for complex network research. Int J Complex Syst. 1695

Doherty IA, Padian NS, Marlow C, Aral SO (2005) Determinants and consequences of sexual networks as they affect the spread of sexually transmitted infections. J Infect Dis 191(Suppl 1):S42-54

Doherty IA, Schoenbach VJ, Adimora AA (2009) Sexual mixing patterns and heterosexual HIV transmission among African americans in the southeastern United States. J Acquir Immune Defic Syndr 52(1):114-120

Doherty IA, Pasquale DK, Liebman AK, Adimora AA, Leone PA (2011) Sexual networks of syphilis and HIV among heterosexuals, MSM, and bisexual men in North Carolina. In: 19th Annual International Society for Sexually Transmitted Diseases Research Meeting 2011 Quebec City Quebec Canada

Doherty IA, Adimora AA, Muth SQ, Serre ML, Leone PA, Miller WC (2011) Comparison of sexual mixing patterns for syphilis in endemic and outbreak settings. Sex Transm Dis 38(5):378-384

Fujimoto K, Flash CA, Kuhns LM, Kim JY, Schneider JA (2018) Social networks as drivers of syphilis and HIV infection among young men who have sex with men. Sex Transm Infect 94(5):365-371. https://doi.org/10.1136/sextrans2017-053288

Hollingsworth TD, Anderson RM, Fraser C (2008) HIV-1 transmission, by stage of infection. J Infect Dis 198(5):687-693

Hurt CB, Beagle S, Leone PA, Sugarbaker A, Pike E, Kuruc J et al (2012) Investigating a sexual network of black men who have sex with men: Implications for transmission and prevention of HIV infection in the United States. J Acquir Immune Defic Syndr 61(4):515-521

Institute of Medicine (US) Committee on the Ryan White CARE Act (2004) Data for Resource Allocation Planning and Evaluation. Measuring what matters: Allocation, planning, and quality assessment for the Ryan white care act. National Academies Press (US), Washington, DC

Jenness SM, Goodreau SM, Rosenberg E, Beylerian EN, Hoover KW, Smith DK et al (2016) Impact of the centers for disease control's HIV preexposure prophylaxis guidelines for men who have sex with men in the United States. J Infect Dis 214(12):1800-1807

Juher D, Saldana J, Kohn R, Bernstein K, Scoglio C (2017) Network-centric interventions to contain the syphilis epidemic in san francisco. Sci Rep 7(1):6464 
Karp G, Schlaeffer F, Jotkowitz A, Riesenberg K (2009) Syphilis and HIV co-infection. Eur J Intern Med 20(1):9-13 Krawczyk CS, Funkhouser E, Kilby JM, Vermund SH (2006) Delayed access to HIV diagnosis and care: special concerns for the southern United States. AIDS Care 18:S35-S44

Liljeros F, Edling CR, Nunes Amaral LA (2003) Sexual networks: implications for the transmission of sexually transmitted infections. Microbes Infect 5(2): 189-196

Mayer KH, Venkatesh KK (2011) Interactions of HIV, other sexually transmitted diseases, and genital tract inflammation facilitating local pathogen transmission and acquisition. Am J Reprod Immunol 65(3):308-316

Napravnik S, Eron Jr JJ, MCKaig RG, Heine AD, Menezes P, Quinlivan E (2006) Factors associated with fewer visits for HIV primary care at a tertiary care center in the southeastern US. AIDS Care 18 Suppl 1: S45-50.

North Carolina HIV/STD/Hepatitis Surveillance Unit (2015a) 2013 North Carolina HIV/STD epidemiologic profile. NC Department of Health and Human Services, Division of Public Health: Raleigh, NC. p 242

North Carolina HIV/STD/Hepatitis Surveillance Unit (2015b) 2014 North Carolina HIV/STD surveillance report. NC Department of Health and Human Services, Division of Public Health: Raleigh, NC. p 98

North Carolina HIV/STD/Hepatitis Surveillance Unit (2020a) 2019 North Carolina HIV surveillance report. North Carolina Department of Health and Human Services, Division of Public Health, Communicable Disease Branch, Raleigh, NC

North Carolina HIV/STD/Hepatitis Surveillance Unit (2020b) 2019 North Carolina sociodemographic characteristics. North Carolina Department of Health and Human Services, Division of Public Health, Communicable Disease Branch, Raleigh, NC

R Core Team (2015) R: A language and environment for statistical computing. 2015, R Foundation for Statistical Computing: Vienna, Austria

Schneider JA, Cornwell B, Ostrow D, Michaels S, Schumm P, Laumann EO et al (2013) Network mixing and network influences most linked to HIV infection and risk behavior in the HIV epidemic among black men who have sex with men. Am J Public Health 103(1):e28-36

Sena AC, Torrone EA, Leone PA, Foust E, Hightow-Weidman L. Endemic early syphilis among young newly diagnosed HIVpositive men in a southeastern U.S. State. AIDS Patient Care STDS, 2008. 22(12): 955-963.

Tieu HV, Liu TY, Hussen S, Connor M, Wang L, Buchbinder S, et al (2015) Sexual networks and HIV risk among black men who have sex with men in 6 U.S. Cities. PLoS One, 10(8): e0134085

USDA Economic Research Service (2013) Rural-urban continuum codes. 10-May-2013 [cited 2020 09-Aug-2020]; Available from: http://www.ers.usda.gov/data-products/rural-urban-continuum-codes.aspx

\section{Publisher's Note}

Springer Nature remains neutral with regard to jurisdictional claims in published maps and institutional affiliations.

\section{Submit your manuscript to a SpringerOpen ${ }^{\circ}$ journal and benefit from:}

- Convenient online submission

- Rigorous peer review

- Open access: articles freely available online

- High visibility within the field

- Retaining the copyright to your article

Submit your next manuscript at $>$ springeropen.com 